\title{
Novel Mutations Identified in the Chinese Han Population with Keratoconus by Next-Generation Sequencing
}

\author{
Binbin Chen, Xiaoning Yu, Xin Zhang, Hao Yang, Yilei Cui, and Xingchao Shentu \\ Eye Center of the Second Affiliated Hospital, School of Medicine, Zhejiang University, \\ Zhejiang Provincial Key Lab of Ophthalmology, Hangzhou, Zhejiang Province, China \\ Correspondence should be addressed to Xingchao Shentu; stxc@zju.edu.cn
}

Received 10 March 2021; Revised 29 August 2021; Accepted 10 December 2021; Published 10 February 2022

Academic Editor: Marco Pellegrini

Copyright (C) 2022 Binbin Chen et al. This is an open access article distributed under the Creative Commons Attribution License, which permits unrestricted use, distribution, and reproduction in any medium, provided the original work is properly cited.

Aim. To identify novel mutations in keratoconus (KC) susceptibility genes in the Chinese Han population. Methods. A total of fifty-two patients with primary $\mathrm{KC}$ were recruited. Blood samples were collected, and genomic DNA was isolated from peripheral blood leukocytes. The entire coding region, intron-exon junctions, and promoter regions of sixteen known KC susceptibility genes were screened with next-generation sequencing technology. All identified variants were further confirmed using the Sanger sequencing technology. The Sorting Intolerant from Tolerant (SIFT), MutationTaster, and PolyPhen 2 programs were used to predict the effect of amino acid substitution on protein. Results. After removing twelve known SNPs (single nucleotide polymorphisms) and three variants predicted to be harmless, nine novel mutations were identified in eight of the fifty-two patients, including c.455C > T:p.P152L in FNDC3B; c.3636_3637del:p.R1212fs in COL4A4; c.5015G > T:p.R1672L, c.3798dupA:p.P1267fs, and c.28G > A:p.A10T in MPDZ; c.1940C > T:p.P647L in DOCK9; c.127_128insGGC:p.Q43delinsRQ in POLG; c.3019G > A: p.V1007I in IPO5; and c.624+ 7-> A in TGFBI. All nine mutations in the patients with KC were heterozygote. Conclusion. This study enlarged the gene profile of $\mathrm{KC}$ and should be further confirmed by well-powered, genome-wide association studies (GWAS) of Han Chinese patients.

\section{Introduction}

Keratoconus $(\mathrm{KC})$ is characterized by bilateral progressive corneal thinning and ectasia [1]. It typically begins in adolescence and progresses until the fourth decade of life [2]. $\mathrm{KC}$ is one of the most common causes of keratoplasty in the developed world [3]. According to epidemiologic studies, the prevalence of KC is approximately 8.8 to 54.4 per 100,000 individuals across the globe, depending on the diagnostic criteria of $\mathrm{KC}$ and population characteristics [3].

Despite the high prevalence of $\mathrm{KC}$, the etiology of the disease is not well understood. Given the fact that $\mathrm{KC}$ is an irreversible disease, it is vital to identify patients with risk factors as soon as possible. Many studies have been performed to identify risk factors related to KC [4-7].

Genetic factors have been shown to contribute to the pathogenesis of $\mathrm{KC}$, although the pathogenesis of many risk genes has yet to be determined [4]. Genome-wide association studies (GWAS) and candidate gene association studies have identified over 150 polymorphisms in more than 60 genes related to increased susceptibility to KC [8]. However, few studies have been conducted in Han Chinese patients with KC. To enlarge the genetic profile of $\mathrm{KC}$ in the Han population, this study was designed to screen the coding regions of NFIB (nuclear factor I B), LIG3 (DNA ligase 3), XRCC1 (X-ray repair cross-complementing 1), TF (transferrin), ZEB1 (zinc finger E-box-binding homeobox 1), NEIL1 (Nei like DNA glycosylase 1), FNDC3B (fibronectin type III domain-containing 3B), COL4A4 (collagen type IV alpha 4 chain), MPDZ (multiple PDZ domain crumbs cell polarity complex component), DOCK9 (dedicator of cytokinesis 9), TGFBI (transforming growth factorbeta induced), PARP1(poly(ADP-ribose) polymerase 1), POLG (DNA polymerase gamma), IPO5 (importin 5), FASLG (Fas ligand), and IL1B (interleukin 1 beta) genes in the Chinese population. 


\section{Methods}

This study adhered to the tenets of the Declaration of Helsinki and the statement on human subjects by the Association for Research in Vision and Ophthalmology. It was approved by the ethics committee of the Second Affiliated Hospital, Medical College of Zhejiang University, Hangzhou, China. Informed consent was obtained from every participant.

Thirty male and twenty-two female $\mathrm{KC}$ patients, ranging from eleven to forty-three years of age, were willing to enroll in this study. All KC patients included in the study had negative family histories for $\mathrm{KC}$. The diagnosis of $\mathrm{KC}$ was made based on clinical manifestations such as corneal stromal thinning, Vogt's striae, Fleischer ring, Munson's sign, conical protrusion of the cornea at the apex, and anterior corneal stromal scar using a slit-lamp microscope, and signs of videokeratography (localized, increased surface power, and/or inferior-superior dioptric asymmetry (Bausch \& Lomb Surgical, Orbtek Inc., Salt Lake City, UT)). Any patient with $\mathrm{KC}$ with a coexisting allergy; atopy $\mathrm{KC}$ secondary to such causes as trauma, LASIK, or other refractive surgeries; or with Ehlers-Danlos syndrome, Down syndrome, osteogenesis imperfecta, or pellucid marginal degeneration were excluded from this research.

Blood samples (five $\mathrm{ml}$ ) of the participants were collected in Vacutainer tubes (Becton Dickinson, Franklin Lakes, NJ) containing ethylene diamine tetraacetic acid. These tubes were preserved at $-80^{\circ} \mathrm{C}$ before genomic DNA was extracted with a Simgen DNA Blood Mini Kit (Simgen, Hangzhou, China) according to the manufacturer's instructions. All patients with $\mathrm{KC}$ underwent mutation screening. For KC patients, all $\mathrm{KC}$ related genes, which were reported in the PubMed database before December 31, 2014, were screened with next-generation sequencing technology (Supplementary Material (available here)), which was based on targeted sequence capturing technology with the SureSelect Target Enrichment Kit (Agilent Technologies, Santa Clara, CA) and Illumina sequencing technology with the HiSeq sequencer (Illumina, San Diego, CA). All the screened variants were compared with the KC-related variants reported in the PubMed database before May 31, 2021.

To avoid false-positive results and to ascertain the significance of the mutations, the following mutations were subsequently confirmed using Sanger sequencing technology: (i) mutations with a minor allele frequency GWAS $<0.1 \%$ (according to data from the May 2012 release of the 1000 Genomes Project and the Single Nucleotide Polymorphism Database); (ii) mutations that were absent from the results of the WES data acquired from 220 Han Chinese individuals without ocular abnormalities (from a commercial database provided by the Genesky Bio-Tech company); (iii) mutations that were never before reported in the PubMed database; and (iv) mutations that were implied as damaged to protein function according to bioinformatics software (as detailed further in this study).

All the coding regions (exons, intron-exon junctions, and promoter regions) of the sixteen genes were amplified with PCR using specific primer sequences. Three different PCR conditions were involved in this study:

(1) Reaction conditions for fragments 1 and 6: the cycling program was $95^{\circ} \mathrm{C}$ for $2 \mathrm{~min} ; 35$ cycles $\times\left(96^{\circ} \mathrm{C}\right.$ for $10 \mathrm{~s}$ and $68^{\circ} \mathrm{C}$ for $1 \mathrm{~min}$ ); and $4^{\circ} \mathrm{C}$ forever

(2) Reaction conditions for fragments 3 to 5 : the cycling program was $95^{\circ} \mathrm{C}$ for $2 \mathrm{~min}$; 11 cycles $\times\left(94^{\circ} \mathrm{C}\right.$ for $20 \mathrm{~s}, 66^{\circ} \mathrm{C}$ minus $0.5^{\circ} \mathrm{C} /$ cycle for $40 \mathrm{~s}$, and $72^{\circ} \mathrm{C}$ for $1 \mathrm{~min}) ; 24$ cycles $\times\left(94^{\circ} \mathrm{C}\right.$ for $20 \mathrm{~s}, 60^{\circ} \mathrm{C}$ for $30 \mathrm{~s}$, and $72^{\circ} \mathrm{C}$ for $1 \mathrm{~min}$ ); $72^{\circ} \mathrm{C}$ for $2 \mathrm{~min}$; and $4^{\circ} \mathrm{C}$ forever

(3) Reaction conditions for fragments 2 and 7: the cycling program was $95^{\circ} \mathrm{C}$ for 2 min; 11 cycles $\times\left(94^{\circ} \mathrm{C}\right.$ for $20 \mathrm{~s}, 62^{\circ} \mathrm{C}$ minus $0.5^{\circ} \mathrm{C} / \mathrm{cycle}$ for $40 \mathrm{~s}$, and $72^{\circ} \mathrm{C}$ for $1 \mathrm{~min}) ; 24$ cycles $\times\left(94^{\circ} \mathrm{C}\right.$ for $20 \mathrm{~s}, 56^{\circ} \mathrm{C}$ for $30 \mathrm{~s}$, and $72^{\circ} \mathrm{C}$ for $1 \mathrm{~min}$ ); $72^{\circ} \mathrm{C}$ for $2 \mathrm{~min}$; and $4^{\circ} \mathrm{C}$ forever

The PCR products were isolated with electrophoresis and sequenced using the BigDye Terminator v3.1 Cycle Sequencing Kit (Applied Biosystems, Foster City, CA) on an Applied Biosystems ABI 3730 Sequencer Analyzer. Finally, the sequencing results were analyzed using the PolyPhred version and compared with the sequences in the NCBI (the National Center for Biotechnology Information) GenBank database.

The SIFT programs were used to predict the effects of mutations on proteins. Based on the theory of evolutionary conservation, amino acid substitution is considered damaging if the SIFT score is $\leq 0.05$; otherwise, the substitution is tolerated. The other two kinds of bioinformatics software-MutationTaster and PolyPhen 2-were used to improve the accuracy of predictability. Both of the predicted scores were positively correlated with pathogenicity, and the significance level was set at 0.85 . Only those that met the following criteria were recognized as harmful to the function of the protein: (i) SIFT score $\leq 0.05$ and (ii) MutationTaster or PolyPhen 2 score $\geq 0.85$.

\section{Results}

The entire coding region, intron-exon junctions, and promoter regions of all involved genes were screened. Twentyfour variants in NFIB, LIG3, XRCC1, TF, ZEB1, NEIL1, FNDC3B, DOCK9, COL4A4, POLG, MPDZ, IPO5, TGFBI, PARP1, FASLG, and IL1B genes were identified in fifty-two sporadic KC patients with next-generation sequencing technology. A total of twenty sequence variants were nonsynonymous single-nucleotide variants; two variants caused frameshift insertion; and two were located in the region of 2 bp around the splicing junction. These results are summarized in Table 1.

The following were excluded from the study: twelve known SNPs (single nucleotide polymorphism) (rs140030018, rs2271980, rs150679929, rs142213781, rs2019 74179, rs201101621, rs200630156, rs201256399, rs181860 632, rs201477273, rs5602117, and rs200401035) and three single nucleotide variants (LIG3, c.1626G $>$ T; ZEB1, c.2998G > C; and PARP1, c.114G > A), whose Sorting Intolerant from Tolerant (SIFT) score was $>0.05$ and PolyPhen 
TABLE 1: All variants identified in KC patients in the present study.

\begin{tabular}{|c|c|c|c|c|c|c|c|c|c|}
\hline Gene & $\begin{array}{l}\text { Nucleotide } \\
\text { change }\end{array}$ & $\begin{array}{l}\text { Amino acid } \\
\text { change }\end{array}$ & SNP ID & $\begin{array}{c}\text { Frequency } \\
\text { (1KG } \\
\text { project) }\end{array}$ & $\begin{array}{l}\text { Gene } \\
\text { region }\end{array}$ & Mutation effect & $\begin{array}{l}\text { SIFT } \\
\text { score }\end{array}$ & $\begin{array}{l}\text { PolyPhen } \\
\text { V2 score }\end{array}$ & MutationTaster \\
\hline NFIB & c. $196 \mathrm{C}>\mathrm{G}$ & p.P66A & rs140030018 & 0.00219649 & Exonic & Nonsynonymous & 0.14 & 0.999 & \\
\hline LIG3 & c. $1626 \mathrm{G}>\mathrm{T}$ & p.K542N & & & Exonic & Nonsynonymous & 0.06 & 0.573 & \\
\hline XRCC1 & c. $1141 \mathrm{G}>\mathrm{A}$ & p.V381M & rs2271980 & & Exonic & Nonsynonymous & 0 & 0.977 & \\
\hline $\mathrm{TF}$ & c. $521 \mathrm{C}>\mathrm{T}$ & p.S174L & rs150679929 & 0.00159744 & Exonic & Nonsynonymous & 0.03 & 0.678 & \\
\hline ZEB1 & c. $2998 \mathrm{G}>\mathrm{C}$ & p.E1000Q & & & Exonic & Nonsynonymous & 0.08 & 0.293 & \\
\hline NEIL1 & c. $833 \mathrm{C}>\mathrm{T}$ & p.T278I & rs142213781 & 0.000599042 & Exonic & Nonsynonymous & 0 & 0.998 & \\
\hline FNDC3B & c. $455 \mathrm{C}>\mathrm{T}$ & p.P152L & & & Exonic & Nonsynonymous & 0 & 0.963 & 1 \\
\hline FNDC3B & c. $1438 \mathrm{G}>\mathrm{A}$ & p.A480T & rs201974179 & 0.000599042 & Exonic & Nonsynonymous & 0.15 & 0.17 & \\
\hline COL4A4 & c.3636_3637 & del:p.R1212fs & & & Exonic & $\begin{array}{c}\text { Frameshift } \\
\text { deletion }\end{array}$ & & & \\
\hline MPDZ & c. $5015 \mathrm{G}>\mathrm{T}$ & p.R1672L & & & Exonic & Nonsynonymous & 0 & & \\
\hline MPDZ & c.3798dupA & p.P1267fs & & & Exonic & $\begin{array}{c}\text { Frameshift } \\
\text { insertion }\end{array}$ & & & \\
\hline MPDZ & c. $394 \mathrm{G}>\mathrm{A}$ & p.G132S & rs201101621 & 0.000399361 & Exonic & Nonsynonymous & 0.03 & & \\
\hline MPDZ & c. $28 \mathrm{G}>\mathrm{A}$ & p.A10T & & & Exonic & Nonsynonymous & 0.05 & & \\
\hline DOCK9 & c. $1940 \mathrm{C}>\mathrm{T}$ & p.P647L & & & Exonic & Nonsynonymous & 0 & & \\
\hline TGFBI & c. $624+7->\mathrm{A}$ & & & & Splicing & & & & \\
\hline PARP1 & c. $2440 \mathrm{~A}>\mathrm{G}$ & p.I814V & rs200630156 & 0.000798722 & Exonic & Nonsynonymous & 0.21 & 0.151 & \\
\hline PARP1 & c. $114 \mathrm{G}>\mathrm{A}$ & p.M38I & & & Exonic & Nonsynonymous & 0.09 & 0.646 & \\
\hline PARP1 & c. $14 \mathrm{C}>\mathrm{G}$ & p.S5W & rs201256399 & 0.00119808 & Exonic & Nonsynonymous & 0 & 0.299 & \\
\hline POLG & c. $3139 \mathrm{C}>\mathrm{T}$ & p.R1047W & rs181860632 & 0.000399361 & Exonic & Nonsynonymous & 0.02 & 1 & \\
\hline POLG & c. $2890 \mathrm{C}>\mathrm{T}$ & p.R964C & rs201477273 & 0.00299521 & Exonic & Nonsynonymous & 0 & 1 & \\
\hline POLG & c.127_128insGGC & p.Q43delinsRQ & & & Exonic & $\begin{array}{l}\text { Frameshift } \\
\text { insertion }\end{array}$ & & & \\
\hline IPO5 & c. $3019 \mathrm{G}>\mathrm{A}$ & p.V1007I & & & Exonic & Nonsynonymous & 0.03 & 0.006 & 0.994 \\
\hline FASLG & c. $280 \mathrm{~T}>\mathrm{G}$ & p.L94V & rs56302117 & 0.00259585 & Exonic & Nonsynonymous & 0 & 0.778 & \\
\hline IL1B & c. $275 \mathrm{C}>\mathrm{T}$ & p.T92I & rs200401035 & 0.000199681 & Exonic & Nonsynonymous & 0.01 & 0.766 & \\
\hline
\end{tabular}

TABle 2: Sequences of primers used in this study.

\begin{tabular}{|c|c|c|c|c|}
\hline Gene & Mutations & Primer name & Fragment size (bp) & Primer sequence \\
\hline FNDC3B & c.455C > T:p.P152L & $\begin{array}{l}6 \mathrm{~F} \\
6 \mathrm{R}\end{array}$ & 417 & $\begin{array}{l}\text { TGCGTTCTTTCCTTTTGTGTT } \\
\text { GCTAGGAATTCCCCATGAGTC }\end{array}$ \\
\hline COL4A4 & c.3636_3637del:p.R1212fs & $\begin{array}{l}7 \mathrm{~F} \\
7 \mathrm{R}\end{array}$ & 324 & $\begin{array}{l}\text { TCCTCATTGCATTTGGAAGGT } \\
\text { GCAACCAGTTGTTGGTGTCTG }\end{array}$ \\
\hline MPDZ & c.5015G > T:p.R1672L & $\begin{array}{l}12 \mathrm{~F} \\
12 \mathrm{R}\end{array}$ & 484 & $\begin{array}{l}\text { GTTCCTTAGTGAGGGGGCTAA } \\
\text { AATGCCTGTTTTTGACCTTCA }\end{array}$ \\
\hline MPDZ & c.3798dupA:p.P1267fs & $\begin{array}{l}9 \mathrm{~F} \\
9 \mathrm{R}\end{array}$ & 494 & $\begin{array}{l}\text { GAGACACAGAGTGGCTGATCC } \\
\text { ACAAACCCACTGCATTCTTTT }\end{array}$ \\
\hline MPDZ & c. $28 \mathrm{G}>$ A:p.A10T & $\begin{array}{l}13 \mathrm{~F} \\
13 \mathrm{R}\end{array}$ & 345 & $\begin{array}{c}\text { CCTGCTTGGGTGAATGATGTC } \\
\text { GGGTCCGGCCTACTGTTTTT }\end{array}$ \\
\hline DOCK9 & c.1940C > T:p.P647L & $\begin{array}{l}17 \mathrm{~F} \\
17 \mathrm{R}\end{array}$ & 353 & $\begin{array}{l}\text { CAAACCTTAAGGGGCTGAGAG } \\
\text { CCTGCAGTAAAACTCCCATCA }\end{array}$ \\
\hline POLG & c.127_128insGGC:p.Q43delinsRQ & $\begin{array}{l}11 \mathrm{~F} \\
11 \mathrm{R}\end{array}$ & 425 & $\begin{array}{c}\text { CAGCTCCACGTCGGGCAAGG } \\
\text { GCCCAAAGCCAGGTGTTCTGACTCC }\end{array}$ \\
\hline IPO5 & c.3019G > A:p.V1007I & $\begin{array}{l}8 \mathrm{~F} \\
8 \mathrm{R}\end{array}$ & 445 & $\begin{array}{l}\text { CCCTCTGTTGCCATTCTGTAA } \\
\text { AGGAAGCACTGTGGAGGAGA }\end{array}$ \\
\hline TGFBI & c. $624+7->\mathrm{A}$ & $\begin{array}{l}19 \mathrm{~F} \\
19 \mathrm{R}\end{array}$ & 402 & $\begin{array}{l}\text { CAGAGTTGCAAGGACCCATCT } \\
\text { GCAAAATGTGGGTTCCACAAG }\end{array}$ \\
\hline
\end{tabular}

V2 (or MutationTaster) score was $<0.85$. The following nine novel mutations were selected for further characterization with Sanger sequencing technology to avoid false-positive results: FNDC3B (c.455C > T:p.P152L), COL4A4 (c.3636_3637del:p.R1212fs), MPDZ (c.5015G > T:p.R1672L;
c.3798dupA:p.P1267fs; and c.28G > A:p.A10T), DOCK9 (c.1940C > T:p.P647L), POLG(c.127_128insGGC:p.Q43delinsRQ), IPO5(c.3019G > A:p.V1007I), and TGFBI (c.624+ $7->A)$. The forward and reverse primers used in the direct polymerase chain reaction (PCR) sequencing are 


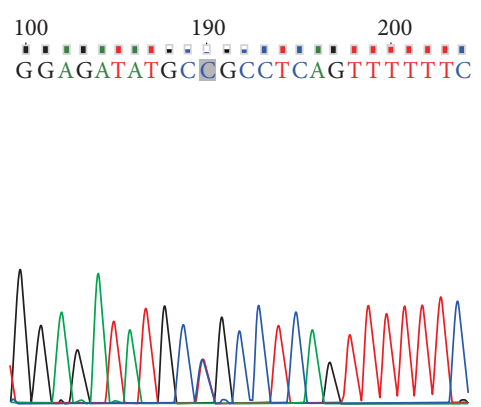

FNDC3c. 455C>T: p. P152L

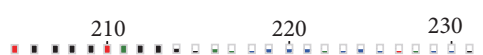

TGGGGTAGGGGAACCCCACCCTACCGGA

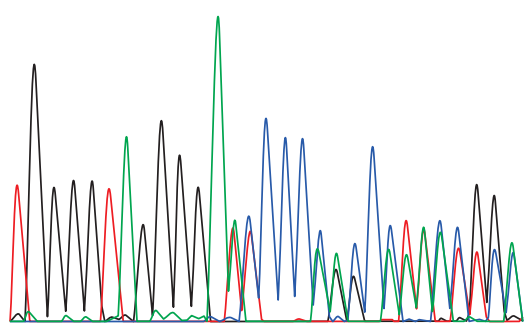

TGFBIc. 127_128i ns GGC: p. Q43del i ns RQ
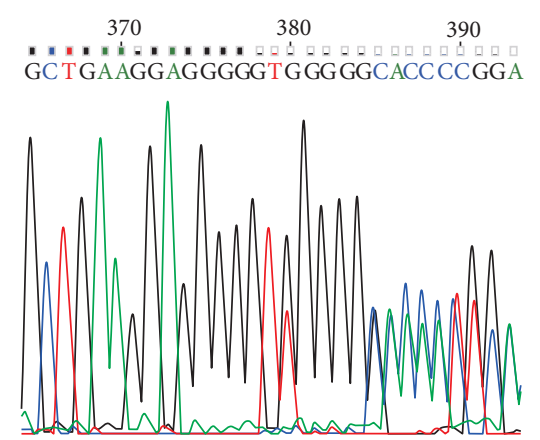

MPDZc. 3798dup A: p. P1267fs

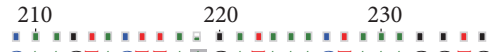

CA AGTACT TAA GATAAACTAAA G G TG

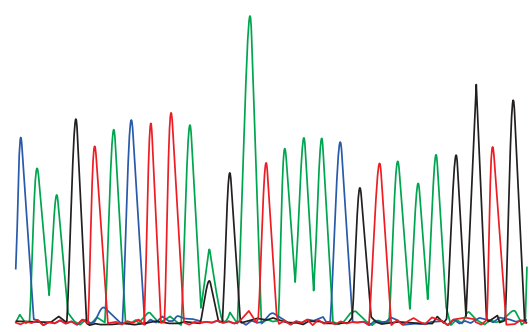

DOCK9c. 1940C>T: p. P647L

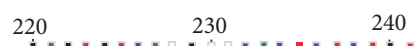

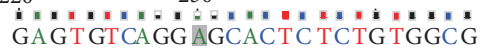

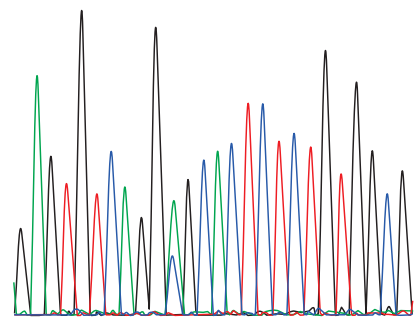

MPDZc. 5015G>T: p. R1672L

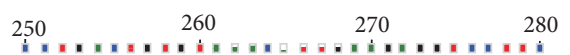

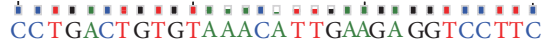

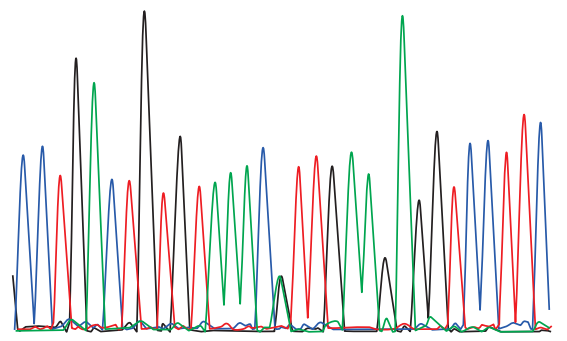

IP05c. $624+7$ - >A
90

100 110

CTCC CA GGGTCTCCTCTCCCCTTTTTTACCOCGTGTT

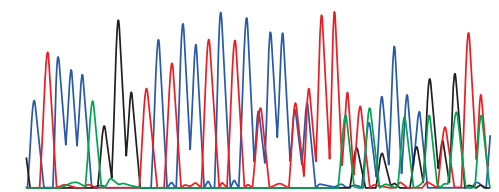

COL4A4c. 3636_3637del: p. R1212fs

180 190 $200 \quad 210$ GCTGCIGAGGCTGCTGTTGCTGCTGCTGCTGCTGCTGCTG̈

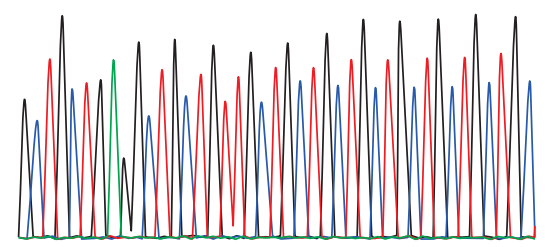

POLGc. 3019G>A: p. V1007I

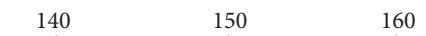

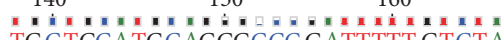

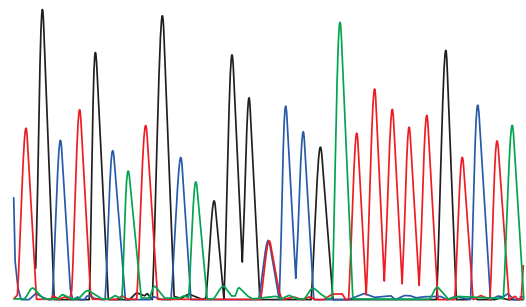

MPDZc. 28G>A: p. A10T

FIGURE 1: Sequence chromatogram of the nine novel mutations.

TABLE 3: The characteristics of 8 patients carrying the nine novel mutations.

\begin{tabular}{|c|c|c|c|c|c|c|}
\hline Subject & Gene & Age at detection & Gender & Eye & Keratoplasty & Central thinnest corneal thickness (OD/OS, $\mu \mathrm{m})$ \\
\hline 1 & $\begin{array}{l}\text { FNDC3B } \\
\text { COL4A4 }\end{array}$ & 24 & Female & $\mathrm{OU}$ & No & $464 / 459$ \\
\hline 2 & IPO5 & 17 & Male & OU & No & $457 / 479$ \\
\hline 3 & MPDZ & 21 & Male & $\mathrm{OU}$ & No & $405 / 370$ \\
\hline 4 & POLG & 23 & Male & OU & No & $459 / 458$ \\
\hline 5 & MPDZ & 16 & Female & OU & No & $426 / 456$ \\
\hline 6 & MPDZ & 32 & Male & $\mathrm{OU}$ & No & $532 / 518$ \\
\hline 7 & $\begin{array}{l}\text { DOCK9 } \\
\text { ZNF469 }\end{array}$ & 17 & Male & OU & No & $414 / 386$ \\
\hline 8 & TGFBI & 24 & Male & OU & No & $502 / 506$ \\
\hline
\end{tabular}

summarized in Table 2. The results indicated that no mutation was identified as a false-positive result with the use of Sanger sequencing technology.

The patient with the mutation FNDC3B was also detected with the mutation COL4A4 (c.3636_3637del: p.R1212fs). In addition, the patient with $\mathrm{KC}$ who carried the mutation DOCK9 (c.1940C > T) was also detected with a ZNF469 mutation (c.3466G > A) (the results of ZNF469 have been published [3]). The SIFT score of the ZNF469 mutation was zero, which is considered to be damaging.

Sequencing chromatograms of the nine novel mutations in the seven genes are shown in Figure 1. All identified 
mutations were located in the exonic region or the region of $2 \mathrm{bp}$ around the splicing junction. According to SIFT and PolyPhen V2 (or MutationTaster) scores, none of the nine mutations was classified as tolerated (Table 1).

Table 3 shows the characteristics of the eight patients with $\mathrm{KC}$ carrying the nine novel mutations. All patients with $\mathrm{KC}$ were diagnosed with bilateral KC. The mean age of the eight patients was twenty-two years old, and six patients were male. Central corneal thickness (CCT) was examined in the $\mathrm{KC}$ patients with videokeratography during their initial visit, and the mean values were $457 \mu \mathrm{m}$ for OD (oculus dexter) and $454 \mu \mathrm{m}$ for OS (oculus sinister).

\section{Discussion}

In the present study, nine novel mutations were identified in FNDC3B (c.455C > T:p.P152L), COL4A4 (c.3636_3637del: p.R1212fs), MPDZ (c.5015G>T:p.R1672L; c.3798dupA: p.P1267fs; and c.28G > A:p.A10T), DOCK9 (c.1940C > T: p.P647L), POLG (c.127_128insGGC:p.Q43delinsRQ), IPO5 (c.3019G > A:p.V1007I), and TGFBI (c.624+7->A) in eight of the fifty-two patients with $\mathrm{KC}$ of Han Chinese ethnicity. The mutation in FNDC3B (c.455C > T:p.P152L) coexisted with the mutation in COL4A4 (c.3636_3637del: p.R1212fs) in one female KC patient (Table 3), and the mutation in DOCK9 (c.1940C > T:p.P647L) coexisted with a potentially damaging ZNF469 (c.3466G > A) mutation in one male KC patient. All of the mutations were found in sporadic $\mathrm{KC}$ cases and were absent in the results of the whole-exome sequencing (WES) data acquired. None of the nine mutations related to $\mathrm{KC}$ have been previously reported.

Since the first case of KC was confirmed in 1854 [9], numerous studies have been conducted to uncover the potential pathophysiology. It should be noted that positive family history was found in $6-20 \%$ of $\mathrm{KC}$ cases [10], and concordance between monozygotic twins was confirmed compared with dizygotic twins, which suggests that genetic factors are dominant components in KC etiology.

As is well known, the human cornea is rich in extracellular matrix (ECM), which maintains corneal transparency and biomechanical strength [11]. Through medical biochemistry techniques - such as immunostaining technology - and proteomics, different abnormalities related to varying risk genes in the expression of ECM components were identified in KC patients [12]. To date, more than ten KC-related genes have been identified as related to ECM component abnormality [12-15]. In the present study, two KC-related mutations were identified in two well-known ECM-associated genes (TGFBI and COL4A4). In KC patients, Bykhovskaya et al. identified a significantly unregulated transcript of the gene TGFBI, which was reported as related to the downregulation of collagen genes (such as COL5A1, coding for collagen V) in the ECM and caused decreased CCT [12]. TGFBI encodes the TGF $\beta$-induced protein and works as a transcription regulator; this extracellular protein can also mediate cell adhesion to collagen, laminin, fibronectin, and proteoglycans [16]. In this study, the coding region of TGFBI was screened, and one novel TGFBI mutation (c.624+7->A) was identified, which further confirmed that TGFBI might be a risk gene for KC patients in China. Collagens are the main components of ECM, and the type IV collagen family (COL4A 1 to COL4A6) consists of the corneal basement membrane [17]. The COL4A4 gene was expressed by the central corneal epithelium. With respect to the function of collagen IV, COL4A4 was also suggested to be a candidate risk gene for KC $[18,19]$. In this study, one COL4A4 (c.3636_3637del: p.R1212fs) that has never been reported in Chinese KC patients was identified.

Recent studies show that part of the genes involved in $\mathrm{KC}$ development are related to CCT. In the present study, FNDC3B and MPDZ are widely thought of as CCT-related genes, and progressive corneal thinning is known to be a feature of the pathophysiology of KC. Lu et al. reported that rs4894535 in FNDC3B and rs1324183 in MPDZ led to decreased CCT with GWAS [20], and the results were replicated in studies by Hao and Sahebjada [21, 22]. In the present study, one novel FNDC3B (c.455C > T:p.P152L) mutation and three MPDZ (c.5015G> T:p.R1672L; c.3798dupA:p.P1267fs; and c.28G > A:p.A10T) mutations were identified in Chinese KC patients; this was consistent with former studies and provided strong genetic evidence that variants in these two genes lead to increased susceptibility to KC. Nevertheless, the role of FNDC3B and MPDZ in $\mathrm{KC}$ remains elusive.

DOCK9 variants were first indicated to be related to KC risk in an Ecuadorian family [23]. Former fundamental studies have shown that DOCK9 could work as an activator of the cell division cycle and regulate the wound-repairing process in the human cornea [23]. With next-generation sequencing technology, this study's KC cohort found one novel mutation (c.1940C > T:p.P647L) that was located in the exon region in DOCK9, and the mutation was predicted to be damaging by SIFT.

IPO5, which encodes a member of the kariopherin superfamily and is involved in protein nuclear transport $[24,25]$, was also screened in our study. One novel mutation (c.3019G > A:p.V1007I) was indicated to be related to KC in this Chinese KC cohort. Consistent with us, Justyna et al. also reported IPO5 mutations in Polish KC patients [26].

We also screened the coding region of POLG, which encodes the only DNA polymerase present in mammalian mitochondria $[27,28]$. Wojcik et al. provided strong evidence that the POLG gene might play a vital role in KC pathogenesis and determining the risk of this corneal disease [29]. However, genetic studies about the relationship between KC and POLG are limited. The results of this study provide new evidence that supports Wojcik's conclusion.

This study had some limitations. First, the limited sample size influenced the significance of the present study. Secondly, just the coding region of the identified genes was screened, while deep intronic substitutions could also induce $\mathrm{KC}$ formation. Thirdly, negative family history indicated that all involved patients were sporadic. Thus, it is difficult to define the genetic pattern of novel mutations. In our view, the absence of these mutations in the majority of Chinese KC patients suggests that their role is not dominant, and the phenotype of the next generation would help define the 
genetic pattern. Fourthly, the present study examined KCrelated genes reported before December 31, 2014, and the additional KC-related genomic loci identified by several genome-wide association studies conducted after that may not have been included, which may have resulted in the incomplete coverage of the loci in this study [30-33].

The exact etiology of KC remains elusive. Our study screened sixteen reported $\mathrm{KC}$ genes and identified nine novel mutations in FNDC3B, COL4A4, MPDZ, DOCK9, POLG, IPO5, and TGFBI, and the results should be further confirmed by well-powered GWAS screening of Han Chinese patients with KC. Further research should be designed to uncover the potential role of the novel mutations in $\mathrm{KC}$ etiology by analysing the corneal characteristics of genetic manipulation in animal models and corneal materials obtained from keratoplasty surgeries of mutation carriers.

\section{Data Availability}

The datasets used and/or analysed during the current study are available from the corresponding author on reasonable request.

\section{Conflicts of Interest}

The authors declare that they have no conflicts of interest.

\section{Acknowledgments}

This research was supported by the National Natural Science Foundation of China (Grant Nos. 81800869, 81970781, 81670834, and 81800807) and the Zhejiang Province Natural Science Foundation of China (Grant Nos. LY17H120002 and LY17H090004).

\section{Supplementary Materials}

Compare with the KC-related mutations reported in the PubMed database from January 1, 2015, to May 31, 2021. (Supplementary Materials)

\section{References}

[1] Y. S. Rabinowitz, "Keratoconus," Survey of Ophthalmology, vol. 42, no. 4, pp. 297-319, 1998.

[2] Y. O. Kok, G. F. Ling Tan, and S. C. Loon, "Review: keratoconus in Asia," Cornea, vol. 31, no. 5, pp. 581-593, 2012.

[3] X. Yu, B. Chen, X. Zhang, and X. Shentu, "Identification of seven novel ZNF469 mutations in keratoconus patients in a Han Chinese population," Molecular Vision, vol. 23, pp. 296-305, 2017.

[4] J. Wheeler, M. A. Hauser, N. A. Afshari, R. R. Allingham, and Y. Liu, "The genetics of keratoconus: a review," Reproductive System \& Sexual Disorders: Current Research, vol. 6, no. 6, 2012.

[5] S. E. M. Lucas, T. Zhou, N. B. Blackburn et al., "Rare, potentially pathogenic variants in 21 keratoconus candidate genes are not enriched in cases in a large Australian cohort of European descent," PLoS One, vol. 13, no. 6, Article ID e0199178, 2018.
[6] Y. S. Rabinowitz, L. Dong, and G. Wistow, "Gene expression profile studies of human keratoconus cornea for NEIBank: a novel cornea-expressed gene and the absence of transcripts for aquaporin 5," Investigative Opthalmology \& Visual Science, vol. 46, no. 4, pp. 1239-1246, 2005.

[7] M. Czugala, J. A. Karolak, D. M. Nowak et al., "Novel mutation and three other sequence variants segregating with phenotype at keratoconus 13q32 susceptibility locus," European Journal of Human Genetics, vol. 20, no. 4, pp. 389-397, 2012.

[8] Y. M. Wang, L. Ma, S. Y. Lu et al., "Analysis of multiple genetic loci reveals MPDZ-NF1B rs1324183 as a putative genetic marker for keratoconus," British Journal of Ophthalmology, vol. 102, no. 12, pp. 1736-1741, 2018.

[9] J. Nottingham, Practical Observations on Conical Cornea, and on the Short Sight, and Other Defects of Vision Connected with it, Liverpool: JohnChurchill; Deighton \& Laughton, London, UK, 1854.

[10] R. H. Kennedy, W. M. Bourne, and J. A. Dyer, "A 48-year clinical and epidemiologic study of keratoconus," American Journal of Ophthalmology, vol. 101, no. 3, pp. 267-273, 1986.

[11] E. M. Feneck, P. N. Lewis, J. Ralphs, and K. M. Meek, "A comparative study of the elastic fibre system within the mouse and human cornea," Experimental Eye Research, vol. 177, pp. 35-44, 2018.

[12] Y. Bykhovskaya, A. Gromova, H. P. Makarenkova, and Y. S. Rabinowitz, "Abnormal regulation of extracellular matrix and adhesion molecules in corneas of patients with keratoconus," International Journal of Keratoconus and Ectatic Corneal Diseases, vol. 5, no. 2, pp. 63-70, 2016.

[13] X. Xu, X. Zhang, Y. Cui et al., "Three novel variants identified within ECM-related genes in Chinese han keratoconus patients," Scientific Reports, vol. 10, no. 1, p. 5844, 2020.

[14] R. Saravani, D. Yari, S. Saravani, and F. Hasanian-Langroudi, "Correlation between the COL4A3, MMP-9, and TIMP-1 polymorphisms and risk of keratoconus," Japanese Journal of Ophthalmology, vol. 61, no. 3, pp. 218-222, 2017.

[15] L. Dudakova, P. Liskova, T. Trojek, M. Palos, S. Kalasova, and K. Jirsova, "Changes in lysyl oxidase (LOX) distribution and its decreased activity in keratoconus corneas," Experimental Eye Research, vol. 104, pp. 74-81, 2012.

[16] C. Engler, S. Chakravarti, J. Doyle et al., “Transforming growth factor-beta signaling pathway activation in keratoconus," American Journal of Ophthalmology, vol. 151, no. 5, pp. 752-759, 2011

[17] X. Bai, D. J. Dilworth, Y.-C. Weng, and D. B. Gould, "Developmental distribution of collagen IV isoforms and relevance to ocular diseases," Matrix Biology, vol. 28, no. 4, pp. 194-201, 2009.

[18] M. Stabuc-Silih, M. Ravnik-Glavac, and D. Glavac, "Polymorphisms in COL4A3 and COL4A4 genes associated with keratoconus," Molecular Vision, vol. 15, pp. 2848-2860, 2009.

[19] S. S. Rong, S. T. U. Ma, X. T. Yu et al., "Genetic associations for keratoconus: a systematic review and meta-analysis," Scientific Reports, vol. 7, no. 1, p. 4620, 2017.

[20] Y. Lu, V. Vitart, K. P. Burdon et al., "Genome-wide association analyses identify multiple loci associated with central corneal thickness and keratoconus," Nature Genetics, vol. 45, no. 2, pp. 155-163, 2013.

[21] X.-D. Hao, P. Chen, Z.-L. Chen, S.-X. Li, and Y. Wang, "Evaluating the association between keratoconus and reported genetic loci in a han Chinese population," Ophthalmic Genetics, vol. 36, no. 2, pp. 132-136, 2015. 
[22] S. Sahebjada, M. Schache, A. J. Richardson et al., "Evaluating the association between keratoconus and the corneal thickness genes in an independent Australian population," Investigative Opthalmology \& Visual Science, vol. 54, no. 13, pp. 8224-8228, 2013.

[23] J. A. Karolak, M. Rydzanicz, B. Ginter-Matuszewska et al., "Variant c.2262A >C in DOCK9 leads to exon skipping in keratoconus family," Investigative Opthalmology \& Visual Science, vol. 56, no. 13, pp. 7687-7690, 2015.

[24] Y.-S. Byun, B. Kang, Y.-S. Yoo, and C.-K. Joo, "Poly(ADPRibose) polymerase inhibition improves corneal epithelial innervation and wound healing in diabetic rats," Investigative Opthalmology \& Visual Science, vol. 56, no. 3, pp. 1948-1955, 2015.

[25] F. Sirchia, E. Di Gregorio, G. Restagno et al., "A case of Feingold type 2 syndrome associated with keratoconus refines keratoconus type 7 locus on chromosome 13q," European Journal of Medical Genetics, vol. 60, no. 4, pp. 224-227, 2017.

[26] J. A. Karolak, P. Polakowski, J. Szaflik, J. P. Szaflik, and M. Gajecka, "Molecular screening of keratoconus susceptibility sequence variants in VSX1, TGFBI, DOCK9, STK24, and IPO5 genes in polish patients and novel TGFBI variant identification," Ophthalmic Genetics, vol. 37, no. 1, pp. 37-43, 2016.

[27] W. C. Copeland and M. J. Longley, "DNA polymerase gamma in mitochondrial DNA replication and repair," The Scientific World Journal, vol. 3, pp. 34-44, 2003.

[28] M. C. De Vries, R. J. Rodenburg, E. Morava et al., "Multiple oxidative phosphorylation deficiencies in severe childhood multi-system disorders due to polymerase gamma (POLG1) mutations," European Journal of Pediatrics, vol. 166, no. 3, pp. 229-234, 2007.

[29] K. Wojcik, E. Synowiec, K. Sobierajczyk et al., "Polymorphism of the DNA base excision repair genes in keratoconus," International Journal of Molecular Sciences, vol. 15, no. 11, pp. 19682-19699, 2014.

[30] H. Choquet, R. B. Melles, J. Yin et al., "A multiethnic genomewide analysis of 44,039 individuals identifies 41 new loci associated with central corneal thickness," Communications Biology, vol. 3, no. 1, p. 301, 2020.

[31] A. J. Hardcastle, P. Liskova, Y. Bykhovskaya et al., "A multiethnic genome-wide association study implicates collagen matrix integrity and cell differentiation pathways in keratoconus," Communications Biology, vol. 4, no. 1, p. 266, 2021.

[32] Y. Hosoda, M. Miyake, A. Meguro et al., "Keratoconus-susceptibility gene identification by corneal thickness genomewide association study and artificial intelligence IBM Watson," Communications Biology, vol. 3, no. 1, p. 410, 2020.

[33] B. J. McComish, S. Sahebjada, Y. Bykhovskaya et al., "Association of genetic variation with keratoconus," JAMA Ophthalmology, vol. 138, no. 2, pp. 174-181, 2020. 